\title{
Reaction, Anticipation and Accent in a Gravitational Pitch Space: Commentary on Ammirante and Thompson
}

\author{
ZOHAR EITAN \\ Tel Aviv University
}

\begin{abstract}
Ammirante and Thompson's intriguing article aims to enhance the ecological validity of their previous findings (Ammirante, Thompson, \& Russo, in press) by using music-like melodic stimuli, rather than random pitch sequences. In line with this aim, I will briefly discuss three issues that may be taken into account in relating the motion-like qualities of melody to music and music-related behavior (finger tapping). First, I suggest how the authors' hypotheses may be examined within a context in which tapping is affected by expectancies for a specific melodic continuation. Second, I discuss how timing and velocity changes associated with melodic distance and contour may give rise to a prototypical joint accent structure, integrating melodic, agogic and dynamic accent. Finally, I note a possible confound of melodic direction and tonality in Ammirante and Thompson's stimuli, and suggest ways to examine the effects of these two dimensions separately.
\end{abstract}

Submitted 2010 August 27; accepted 2010 September 11.

\section{REACTION AND ANTICIPATION}

WHILE periodic movement to music, finger tapping included, may stem from reactions to musical events just heard (the focus of Ammirante and Thompson's study), it may also be affected by anticipating forthcoming events (Pecenka \& Keller, 2009). Anticipation (based on veridical memory) may be explicit and precise for a listener or performer who knows the specific music in question well. For those attending to or performing novel music which exhibits perceptible regularities, or novel music in a style they know well, anticipation (based on schematic memory) may be implicit and probabilistic, as event and eventsuccession probabilities may generate expectations concerning forthcoming events (Bharucha, 1984; Huron, 2006).

While Ammirante and Thompson's hypotheses, and most of their analysis, address reactions to just-heard auditory events, their theoretical framework could be applied to anticipated events as well. The authors suggest that overlap of auditory and motor representations associates contour change with change in the direction of bodily motion, and pitch distance (melodic intervals) with spatial distance. Inter-tap intervals (ITI) and tap velocity (TV) would then be affected by pitch contour and pitch intervals in ways that reflect this analogy: slowdown would follow points of contour change, as compared to contour continuation (as the tapper reacts to the deceleration of movement implied by the change of contour), and acceleration and deceleration, respectively, would follow increase and decrease in pitch distance. Yet, for an actively moving human agent, anticipating a change in direction, or anticipating a need to traverse a longer distance within a set time, may trigger timing and velocity changes. Indeed, the motor-auditory analogy underlying the article seems to suggest an anticipatory, rather than reactive motion. For instance, a positive correlation between pitch distance and velocity implies acceleration of motion when pitch distance increases (e.g., between D and C in the sequence E flat - D - C, as in Fig. 2, Seq. Pos. 1-3, p. 97), rather than following that increase. Similarly, the slowing down generated by contour change would occur between notes C and D in the sequence E flat - D - C - D (Fig. 2, Seq. Pos. 3-4), where the change of direction actually takes place, rather than following this change. However, such effects could materialize only if the listener-tapper indeed develops expectations, either explicitly, by way of recognition of the specific melodic stimulus, or implicitly, by perceiving regularities within the stimulus or by associating it with probabilities learned through exposure to other music. 
Such expectations could be experimentally examined, within the framework of a continuation tapping paradigm, in various ways. The simplest would be through veridical memory: making sure participants learn and can recognize the musical stimuli (the melodic sequence to be generated by tapping) prior to the experiment itself. In another design, involving musically-trained participants, a moving window showing (in staff notation) both the currently-heard note (generated by the current tap) and the forthcoming one may be presented to participants (note, however, that in this paradigm visual presentation may affect the examined auditory-motor relationships). Expectations may also be generated by creating regularities within the melodic stimulus itself, such as a constant metrical structure or repeated melodic or rhythmic patterns (e.g., a melodic sequence such as G-C-B A-D-C B-E-D . . .), generating higher-level patterning. Finally, as noted, expectations may be generated by clearly associating the melodic stimulus to the constraints and probabilities of a known body of music, such as the constraints of Western major-minor tonality.

In Ammirante and Thompson's study, multiple exposures to each stimulus notwithstanding, stimulus structure does not facilitate the creation of veridical memory representations; (and indeed, learning effects that would indicate such representations were not found; see "preliminary analysis," p. 98), nor does it enhance the evocation of long-term schematic representations. As the authors note, their stimulus construction aims to minimize expectancies based on higher-order rhythmic or melodic patterning ("stimuli and procedure," p. 96). Minimal contrasts within and between stimuli further hinder clear distinctions, and thus hamper recognition: all stimuli are transformations (retrograde or transposed inversion) of two sequences; they employ only stepwise progressions and equi-durational rhythm, and (as indeed suggested by the autocorrelation analysis, p. 98) they do not generate a clear metrical structure. Furthermore, tonal patterning is also weak and ambiguous. Though the pitch repertory is limited to two diatonic collections (the lower pentachords of $\mathrm{C}$ major and $\mathrm{C}$ minor scales), this constraint alone does not ensure an unambiguous tonality, since these collections are also segments of other major or minor scales, such as $\mathrm{F}$ major or A minor (for the "C major" pentachord), E-flat major or $\mathrm{G}$ minor (for the "C minor" pentachord). Thus, for instance, nothing in melodies 1 and 5 (Fig. 1, p. 96) suggests that they are in $\mathrm{C}$, rather than $\mathrm{F}$ major.

By carefully hindering veridical memory and minimizing top-down expectancies Ammirante and Thompson create a controlled environment that better monitors the reactive effects they wish to investigate. This, however, calls for complementary experiments in which the proposed overlap of motor and pitch representations would be examined, using continuation tapping or other paradigms, in the framework of an expectancy-based model. While results of previous studies of expressive timing are clearly relevant, more work examining (and disentangling) the effects of veridical and schematic expectancies on the motion-like implications of pitch contour and pitch distance in a controlled, yet music-like environment is called for.

\section{PITCH MOTION AND THE INTERACTION OF MELODIC, AGOGIC AND DYNAMIC ACCENTS}

While the nature of melodic accent is somewhat equivocal (Huron \& Royal, 1996), there is little dispute as to the importance of two other types of "phenomenal" accent: dynamic accent, assigned to a tone louder than its surroundings, and agogic (durational) accent, assigned to a tone when the time interval between its onset and that of the following tone is longer than the preceding and following inter-onset intervals (Steedman, 1977; Tekman, 2002).

In a tapping task, a longer ITI (like a longer IOI in a melodic stimulus) would enhance agogic accent on the tone initiating it. Accelerated flexion TV would generate higher impact intensity, and hence (in normal circumstances; in Ammirante and Thompson's experiment, intensity was kept constant regardless of TV) a dynamic accent at the tone (or tap) terminating finger flexion. In contrast, decelerated TV would result in reduced loudness, and hence the preceding, louder tone would receive a dynamic accent.

Assuming an anticipation-based (rather than reactive) action, the effects of pitch distance and pitch contour on ITI and TV, respectively, would generate a joint accent, bringing together contour pivots and melodic interval accent with dynamic and agogic accents. Consider, for instance, a contour change (CC) three-note pattern, in which pitch distance between the initial and medial tone is larger than that between the medial and final tone (e.g., C4-F4-E4). If responses reflect anticipation, the ITI between the initial and medial tone (where pitch distance is greater) would be shorter than that between the medial and terminal tone, creating an agogic accent on the medial tone. In addition, $\mathrm{CC}$ would generate a slower TV toward the 
terminal tone, hence decreased loudness. The preceding medial tone would thus receive not only agogic accent, but a dynamic accent as well. Thus, the motor-auditory analogy suggested by Ammirante and Thompson may serve to elucidate congruence between melodic, agogic, and dynamic accents -- though only if anticipatory action is assumed.

Importantly, the relationships between contour, pitch distance, onset interval and loudness proposed here reflect both perceptual data and statistical data generated from diverse musical repertories. Thus, for instance concurrence of melodic accents (contour pivots) and durational accents increases accent salience (Pfordresher, 2003), and contour pivots are often mistaken for increases in loudness (Repp, 2010). Correspondingly, Eitan (1996) shows that in different musical repertories, both tonal (Chopin) and posttonal (Alban Berg), melodic peaks tend to receive agogic and dynamic accents, and are typically preceded by relatively large melodic intervals.

Note that an anticipatory action based on the motor-auditory coupling suggested by Ammirante and Thompson would suggest, in line with Huron and Royal (1996) and Thomassen (1992), and contrary to the stance taken by Ammirante and Thompson themselves, that it is the tone initiating CC (the contour pivot, e.g., the medial tone in Fig. 7, left), rather that the tone following it (the terminal tone in Fig. 7, left) that is accented -- not only melodically, but dynamically as well, regardless of any implications of meter.

\section{CONTROLLING FOR THE EFFECTS OF TONALITY AND CONTOUR}

An interesting finding of this study was the negative correlation between tap velocity (TV) and pitch height. The authors suggest that this result stems from the "pull" of C, the tonal center. This interpretation, however, is problematic, and not only because (as suggested above) the stimuli do not unequivocally suggest $\mathrm{C}$ as a tonal center. Even if $\mathrm{C}$ would have been a clear tonal center, stimulus design would involve two confounding variables, melodic direction and tonality, since the (assumed) tonal center is always the lowest tone in the sequence. An alternative (or complementary) interpretation of the above finding, consistent with the authors' motor-auditory overlap hypothesis, would be based on mapping pitch motion onto motion in the vertical plane, controlled by gravity. Thus, pitch motion "down" -- supported by gravity -- would accelerate, while motion "up" (opposing gravity) would decelerate. Indeed, Larson (e.g., 2004) suggests a model mapping physical motion onto pitch motion, in which the pull of a tonal center (termed "magnetism") and a pull downwards ("gravity"), together with "inertia" (a tendency to continue in the same direction) are proposed as independent "forces." Controlling for possible effects of tonal "magnetism" and directional "gravity," and examining whether they indeed affect the velocity and timing of music-related motion may enhance Ammirante and Thompson's stimulating mapping of melodic progression and bodily motion.

\section{REFERENCES}

Ammirante, P., Thompson, W. F., \& Russo, F. A. (In Press). Ideomotor effects of pitch in continuation tapping. Quarterly Journal of Experimental Psychology.

Bharucha, J. J. (1984). Event hierarchies, tonal hierarchies, and assimilation: a reply to Deutsch and Dowling. Journal of Experimental Psychology: General, Vol. 63, pp. 421-5.

Eitan, Z. (1997). Highpoints: A Study of Melodic Peaks. Philadelphia: University of Pennsylvania Press.

Huron, D. (2006). Sweet Anticipation: Music and the Psychology of Expectation. Cambridge, MA: MIT Press.

Huron, D., \& Royal, M. (1996). What is melodic accent? Converging evidence from musical practice. Music Perception, Vol. 13, No. 4, pp. 489-516.

Larson, S. (2004). Musical forces, melodic expectation: Comparing computer models and experimental results. Music Perception, Vol. 21, No. 4, pp. 457-499.

Pecenka, N., \& Keller, P. E. (2009). Auditory pitch imagery and its relationship to musical synchronization. Annals of the New York Academy of Sciences, Vol. 1169, No. 1, pp. 282-286. 
Pfordresher, P. Q. (2003). The role of melodic and rhythmic accents in musical structure. Music Perception, Vol. 20, No. 4, pp. 431-464.

Repp, B. H. (2010). Do metrical accents create illusory phenomenal accents? Attention, Perception, \& Psychophysics, Vol. 72, No. 6, pp. 1390-1403.

Steedman, M.J. (1977). The perception of musical rhythm and metre. Perception, Vol. 6, No. 5, pp. 555-569.

Tekman, H. G. (2002). Perceptual integration of timing and intensity variations in the perception of musical accents. Journal of General Psychology, Vol. 129, No. 2, pp. 181-191.

Thomassen, J. (1982). Melodic accent: Experiments and a tentative model. Journal of the Acoustical Society of America, Vol. 71, No. 6, pp. 1596-1605. 